\title{
An Improved Method for 2-D Self-Similar Image Synthesis
}

\author{
Lance M. Kaplan, Member, IEEE, and C.-C. Jay Kuo, Senior Member, IEEE
}

\begin{abstract}
In this paper, we propose a new method called incremental Fourier synthesis to generate 2-D self-similar images based on a 2-D fractional Browninan motion $(\mathrm{fBm})$ model. With this method, the stationary increments of $\mathrm{fBm}$ are created by a Fourier synthesis method and the increments are added up to generate the nonstationary 2-D fBm process. Since the new method takes advantage of the FFT, its computational complexity is only $O\left(N^{2} \log _{2}(N)\right)$, and its memory requirement is only $O\left(N^{2}\right)$ for a self-similar image of size $N \times N$.
\end{abstract}

\section{INTRODUCTION}

$\mathbf{F}$ RACTIONAL Brownian motion ( $\mathrm{fBm}$ ) is a useful nonstationary stochastic model for describing many natural phenomena with a self-similar property [9]. In computer graphic applications, the generation of $2-\mathrm{D} \mathrm{fBm}$ realizations is used to create natural-looking landscapes and clouds [8], [11]. The statistical self-similar property of fBm is a key to the natural-looking textured surface where the "roughness" of the surface is invariant to the scale and the increments are stationary. For some computer graphic applications, the exact roughness-invariant property is not crucial in generating natural-looking images, and as a result, methods loosely based on the statistics of $\mathrm{fBm}$ can be used to generate images with an approximate self-similar property. The two most common methods are midpoint displacement and Fourier synthesis [11]. Other methods include more sophisticated variants of midpoint displacement [7], [12], [13], linear filtering [1], wavelet synthesis [13], and procedura1 textures [10].

The midpoint displacement is the fastest fBm synthesis method with a complexity of $O\left(N^{2}\right)$, where $N^{2}$ is the size of the image. The method uses simple recursive rules over scales analogous to deterministic fractals. However, midpoint displacement fails to create processes with stationary increments and suffers from creasing [8]. The Fourier synthesis method tries to recreate the $1 / f$ spectrum via fast Fourier transform (FFT) with a computational complexity of $O\left(N^{2} \log _{2}(N)\right)$, but the method fails to consider the aliasing effect of a finite lattice. Moreover, Fourier synthesis actually renders a stationary process. Wavelet synthesis generates nearly $1 / f$

Manuscript received June 20, 1994; revised October 2, 1995. This work was supported by the National Science Foundation Presidential Faculty Fellow (PFF) Award ASC-9350309. The associate editor coordinating the review of this manuscript and approving it for publication was Dr. Fredrick Minitzer.

L. M. Kaplan is with the Radar and Communications Systems Segment, Hughes Aircraft Company, Los Angeles, CA 90009 USA (e-mail: Imkaplan@msmail4.hac.com).

C.-C. J. Kuo is with the Signal and Image Processing Institute and the Department of Electrical Engineering Systems, University of Southern California, Los Angeles, CA 90089 USA (e-mail: cckuo@sipi.usc.edu).

Publisher Item Identifier S 1057-7149(96)03173-9. approximations to $\mathrm{fBm}$ that have uncorrelated wavelet coefficients [14]. These nearly $1 / f$ processes, however, do not even have stationary increments. Finally, linear filtering methods approximate the power spectrum of $\mathrm{fBm}$ using only a finite number of poles, and these methods are unable to entirely capture the persistence of $\mathrm{fBm}$. By persistence, we mean the long-term correlation of $\mathrm{fBm}$, which provides an infinite pole at the origin in the frequency domain.

While the above-mentioned synthesis methods do not generate exact $\mathrm{fBm}$ samples, each method does have specific strengths and weaknesses for applications such as computer graphics and turbulence modeling. A discussion about the utility of various $\mathrm{fBm}$-based synthesis techniques for landscape generation can be found in [10]. This paper, on the other hand, focuses on a method to generate processes with almost perfect fBm statistics at a reasonable computational load. For some applications, it is important for the textures to be synthesized based on the exact statistics of fBm. For instance, an artist may want the roughness-invariant property of a texture to hold as a user zooms in to the texture. In other cases, one needs to use 2-D $\mathrm{fBm}$ images as test-case images.

When the generation of true $\mathrm{fBm}$ is important, one could resort to very computationally complex methods. Höfer [2] et. al used the Cholesky decomposition to generate exact 2-D $\mathrm{fBm}$ realizations. Although the Cholesky method does generate processes with true $\mathrm{fBm}$ statistics, the algorithm suffers from a computational complexity of $O\left(N^{6}\right)$ and requires a $N^{2} \times N^{2}$ matrix, i.e., a memory complexity of $O\left(N^{4}\right)$, to generate an image of size $N \times N$. The high computational load and memory requirements make the Cholesky method impractical for synthesis of even moderate-size images.

In this work, we propose a new method called incremental Fourier synthesis to generate 2-D $\mathrm{fBm}$, where a periodic random field is created by Fourier synthesis such that the field's statistics closely match those of $\mathrm{fBm}$ increments over half the field's spatial period. The increments are then added up to generate the nonstationary $2-\mathrm{D} \mathrm{fBm}$ process. Since the new method takes advantage of the FFT, its computational complexity is only $O\left(N^{2} \log _{2}(N)\right)$, and its memory requirement is only $O\left(N^{2}\right)$. Moreover, the method can generate processes whose statistics match almost perfectly to true $\mathrm{fBm}$ statistics.

\section{Fractional Brownian Motion}

$\mathrm{FBm}$ is a random process that was popularized by Mandelbrot and Van Ness [9]. In this paper, we consider the straightforward generalization on $1-\mathrm{D} \mathrm{fBm}$ signals to 2-D fBm images. A 2-D fBm process is a mean-zero Gaussian process 
$B\left(t_{x}, t_{y}\right)$ satisfying

$$
B(0,0)=0
$$

$$
\operatorname{VAR}\left[B\left(t_{x}+r_{x}, t_{y}+r_{y}\right)-B\left(t_{x}, t_{y}\right)\right]=f\left(r_{x}, r_{y}\right) \sigma^{2}
$$

where $\sigma^{2}=\operatorname{VAR}\left[B\left(s_{x}+1, s_{y}\right)-B\left(s_{x}, s_{y}\right)\right]$

$$
f\left(r_{x}, r_{y}\right)=\tilde{f}\left(\left\|\left(r_{x}, r_{y}\right)^{T}\right\|\right)=\left(\sqrt{r_{x}^{2}+r_{y}^{2}}\right)^{2 H}
$$

and $0<H<1$. Equations (2) and (3) constitute the 2-D self-similarity condition. It implies that the variance of any increment is independent of orientation and dependent only on the length of the increment. The parameter $H$ is known as the Hurst parameter, and each realization of $2-\mathrm{D} \mathrm{fBm}$ is a fractal with a dimension of $D=3-H$ [11].

In this paper, we want to generate samples of $2-\mathrm{D} \mathrm{fBm}$ on a discrete grid. One can show from (1) and (2) that 2-D fBm is a nonstationary isotropic process whose correlation function for a sampling period of $\Delta x$ in both $x$ and $y$ directions is

$$
\begin{aligned}
r_{B} & \left(m_{x}, m_{y} ; n_{x}, n_{y}\right) \\
= & E\left[B\left(\Delta x m_{x}, \Delta x m_{y}\right) B\left(\Delta x n_{x}, \Delta x n_{y}\right)\right] \\
= & \frac{\sigma^{2}}{2}|\Delta x|^{2 H}\left[f\left(m_{x}, m_{y}\right)+f\left(n_{x}, n_{y}\right)\right. \\
& \left.-f\left(n_{x}-m_{x}, n_{y}-m_{y}\right)\right] .
\end{aligned}
$$

Note that the shape of the correlation of discrete 2-D fBm is invariant to the chosen sampling rate or scale. This form of scale invariance is a direct result of the self-similarity condition. As a result, the textured appearance of $2-\mathrm{D} \mathrm{fBm}$ sampled every $\Delta x$ units and scaled by a factor of $|\Delta x|^{-H}$ is identical to the appearance of $\mathrm{fBm}$ sampled every one unit. Without loss of generality, we set $\Delta x=1$. We define the first-order discrete increments of 2-D $\mathrm{fBm}$ as

$$
\begin{aligned}
& I_{x}\left(m_{x}, m_{y}\right)=B\left(m_{x}+1, m_{y}\right)-B\left(m_{x}, m_{y}\right), \text { and } \\
& I_{y}\left(m_{x}, m_{y}\right)=B\left(m_{x}, m_{y}+1\right)-B\left(m_{x}, m_{y}\right) .
\end{aligned}
$$

The second-order increments of 2-D $\mathrm{fBm}$ are defined as

$$
\begin{aligned}
& I_{2}\left(m_{x}, m_{y}\right)=I_{x}\left(m_{x}, m_{y}+1\right)-I_{x}\left(m_{x}, m_{y}\right) \\
& =I_{y}\left(m_{x}+1, m_{y}\right)-I_{y}\left(m_{x}, m_{y}\right) \\
& =B\left(m_{x}+1, m_{y}+1\right)+B\left(m_{x}, m_{y}\right) \\
& -B\left(m_{x}+1, m_{y}\right)-B\left(m_{x}, m_{y}+1\right) .
\end{aligned}
$$

Note that samples of $\mathrm{fBm} B\left(m_{x}, m_{y}\right)$ can be calculated for $m_{x} \geq 0$ and $m_{y} \geq 0$ by using the values of the second-order increments and the first-order increments along the $x$ and $y$ axes. The first and second-order increments are stationary. The correlation functions of these increments are

$$
\begin{aligned}
& r_{x}\left(m_{x}, m_{y}\right) \\
& =\frac{\sigma^{2}}{2}\left[f\left(m_{x}+1, m_{y}\right)+f\left(m_{x}-1, m_{y}\right)-2 f\left(m_{x}, m_{y}\right)\right] \\
& r_{y}\left(m_{x}, m_{y}\right) \\
& \left.=\frac{\sigma^{2}}{2} f\left(m_{x}, m_{y}+1\right)+f\left(m_{x}, m_{y}-1\right)-2 f\left(m_{x}, m_{y}\right)\right]
\end{aligned}
$$

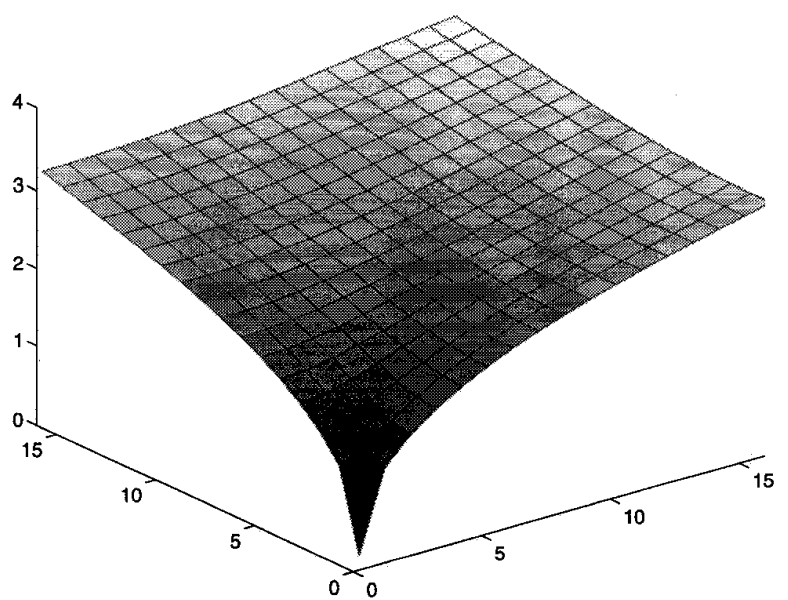

(a)

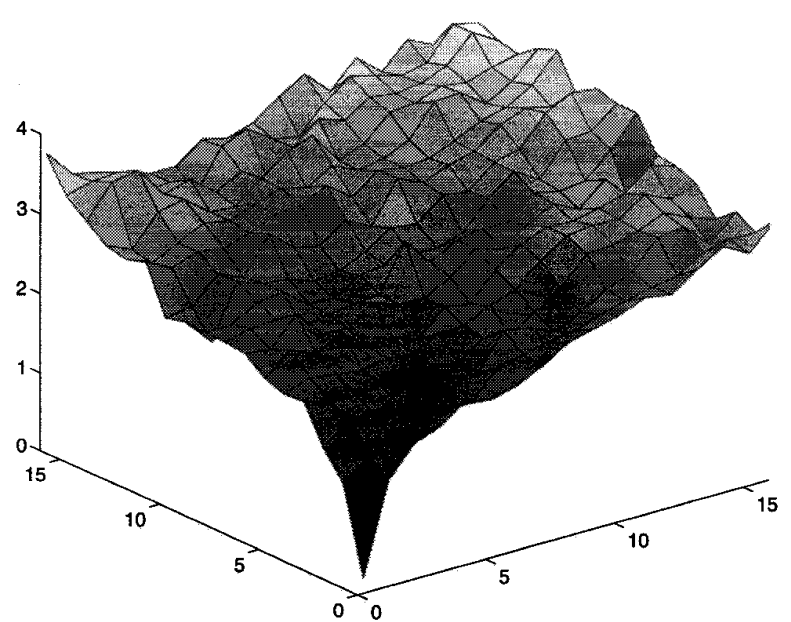

(b)

Fig. 1. Variance of the generated $\mathrm{fBm}$ images for $H=0.2$ : (a) Theoretical; (b) experimental.

$$
\begin{aligned}
& r_{2}\left(m_{x}, m_{y}\right) \\
& =\frac{\sigma^{2}}{2}\left[2 \left(f\left(m_{x}+1, m_{y}\right)+f\left(m_{x}-1, m_{y}\right)\right.\right. \\
& \left.\quad+f\left(m_{x}, m_{y}+1\right)+f\left(m_{x}, m_{y}-1\right)\right) \\
& \quad-\left(f\left(m_{x}+1, m_{y}+1\right)+f\left(m_{x}+1, m_{y}-1\right)\right. \\
& \quad+f\left(m_{x}-1, m_{y}+1\right) \\
& \left.\left.\quad+f\left(m_{x}-1, m_{y}-1\right)\right)-4 f\left(m_{x}, m_{y}\right)\right] .
\end{aligned}
$$

The above correlations are discrete functions. Their Fourier transforms are periodic, and it is, in general, difficult to compute these transforms analytically. However, by treating each correlation to be a continuous function, we can compute the Fourier transform more easily and observe some important properties of the actual periodic spectrum. By using the fact [3] that

$$
\begin{aligned}
& \int_{-\infty}^{\infty} \int_{-\infty}^{\infty}{\sqrt{t_{x}^{2}+t_{y}^{2}}}^{2 H} e^{-j\left[t_{x} \omega_{x}+t_{y} \omega_{y}\right]} d t_{x} d t_{y} \\
& =-\frac{2 \sqrt{\pi} \Gamma(2 H+2) \sin (\pi H)}{{\sqrt{\omega_{x}^{2}+\omega_{y}^{2}}}^{2 H+2}}
\end{aligned}
$$


and the "shifting" property of the Fourier transform, one can derive the Fourier transform of (8) (when integers $m_{x}$ and $m_{y}$ are replaced by real variables $t_{x}$ and $t_{y}$ ) to be

$$
\begin{aligned}
& S_{2}\left(\omega_{x}, \omega_{y}\right) \\
& =\frac{32 \sqrt{\pi} \sin ^{2}\left(\omega_{x} / 2\right) \sin ^{2}\left(\omega_{y} / 2\right) \Gamma(2 H+2) \sin (\pi H)}{{\sqrt{\omega_{x}^{2}+\omega_{y}^{2}}}^{2 H+2}} .
\end{aligned}
$$

One must consider an aliased version of (9) due to the sampling of the second-order increment. As $\omega_{x}$ and $\omega_{y}$ go to zero at the same rate, (9) can be written as

$$
S_{2}(\omega, \omega) \approx C \frac{\omega^{4}}{\omega^{2 H+2}}
$$

and since $0<H<1$, one can say that the spectrum of the continuous increments has a value of zero at the origin (i.e., $\omega_{x}=\omega_{y}=0$ ). Moreover

$$
S_{2}\left(2 \pi k_{x}, 2 \pi k_{y}\right)=0, \quad \forall k_{x}, k_{y} \in \mathbf{Z} .
$$

Equations (9) and (10) indicate that the periodic spectrum of the sampled increments (sampled at intervals of one unit) has values of zeros whenever $\omega_{x}=0$ or $\omega_{y}=0$ and does not approach zero at any other points. The importance of this spectral property will be seen in Section IV.

\section{PERIODIC RANDOM FIELdS}

To describe the synthesis algorithm, we must first discuss some properties of periodic stationary Gaussian random fields (PSGRF). A PSGRF has a correlation function that satisfies

$$
R\left(m_{x}+k N, m_{y}+l N\right)=R\left(m_{x}, m_{y}\right), \quad \forall k, l \in \mathbf{Z} .
$$

Each realization of this random field is also periodic with a period of $N$ in both the $x$ and $y$ directions. Thus, it is only necessary to know the values of the field over an $N \times N$ lattice of points, and the correlation function only needs to be considered for time lags that lie on an $N \times N$ grid. Due to symmetry of the correlation function about the origin, we have

$$
R\left(m_{x}, m_{y}\right)=R\left(N-m_{x}, N-m_{y}\right) .
$$

For cases where the correlation function is symmetric around both the $x$ and $y$ axes, i.e.

$$
\begin{aligned}
& R\left(m_{x}, m_{y}\right)=R\left(N-m_{x}, m_{y}\right) \text { and } \\
& R\left(m_{x}, m_{y}\right)=R\left(m_{x}, N-m_{y}\right)
\end{aligned}
$$

the correlation function can be uniquely defined by lags with $m_{x}, m_{y}=0, \cdots, N / 2$. The other values of the correlation function can be determined through (12) and (13).

The importance of periodic random fields is due to the fact that 2-D DFT is the Karhunen-Loéve transform (KLT) for such fields. A nice result of this property is that realizations of periodic random fields are easy to generate because one just needs to scale white noise by the square root of the field's power spectrum and then calculate the inverse 2-D DFT. In fact, this generation procedure is used in normal Fourier synthesis of $\mathrm{fBm}$ where the power spectrum is assumed to be

$$
\hat{R}\left(k_{x}, k_{y}\right)=C /{\sqrt{k_{x}^{2}+k_{y}^{2}}}^{2 H+2} \quad \forall k_{x}, k_{y}=0, \cdots, N / 2 .
$$

The other values- of the power spectrum are determined by symmetrically expanding $\hat{R}\left(k_{x}, k_{y}\right)$. Usually, the first $N / 2 \times$ $N / 2$ values of the generated field are taken as the $\mathrm{fBm}$ image in order to avoid artifacts from the periodicity of the field.

\section{INCREMENTAL FOURIER SYNTHESIS}

The idea to create samples of $\mathrm{fBm}$ over an $(M+1) \times$ $(M+1)$ grid is to generate the stationary increments over an $M \times M$ grid. We exploit the fact that the DFT represents the KLT for a PSGRF. Ideally, we generate an $N \times N$ PSGRF (where $N=2 M$ ) whose correlation function over the region $[-M, M] \times[-M, M]$ matches the correlation of the $\mathrm{fBm}$ increments. The synthesis is accomplished by scaling white noise by the square root of the desired power spectrum (i.e., the DFT of the correlation function) and then applying the inverse DFT. Next, we cut an $M \times M$ segment out from the PSGRF so that the correlation function for the segment matches the true $\mathrm{fBm}$ increment correlations for lags $\left(l_{x}, l_{y}\right) \in$ $[-M, M] \times[-M, M]$. In addition, the $M \times M$ segment avoids the wraparound effect of the periodic correlation function for the PSGRF.

Unfortunately, a few problems arise in the above synthesis procedure that limits the creation of exact $\mathrm{fBm}$ increments. One problem to consider is that the target (or desired) periodic correlation function may not be positive definite. This could happen because periodically extending a truncated correlation function is equivalent to convolving the actual power spectrum by a sinc and then sampling to create the power spectrum of the periodic random field. Due to the Gibbs phenomenon, some of the values of the DFT of the periodic correlation function may be negative. By considering these bad values to be zero, we create the actual power spectrum that generates the increments. Because the negative values will occur near frequencies where the original power spectrum is zero, the difference between actual and target correlation functions will be small. Another point to consider is that the first- and second-order increments cannot be generated independently or else major creasing will appear. The dependence of $I_{2}\left(m_{x}, m_{y}\right), I_{x}\left(m_{x}, m_{y}\right)$, and $I_{y}\left(m_{x}, m_{y}\right)$ is due to (5), where the subtraction is taken modulo $N$. In fact, by taking the DFT of (5), we see that the DFT coefficients of the first-order increments when the frequencies are nonzero (i.e., $k_{x}>0, k_{y}>0$ ) are completely determined by the corresponding DFT coefficients of the second-order increments. Moreover, the DFT coefficients for $I_{2}\left(m_{x}, m_{y}\right)$ must be zero for the zero frequencies (i.e. $k_{x}=0$ or $\left.k_{y}=0\right)$, and thus, the actual power spectrum of $I_{2}\left(m_{x}, m_{y}\right)$ is forced to zero at the zero frequencies. Because the power spectrum of the nonperiodic field is zero at the zero frequencies (see Section II), the change to the power spectrum will not greatly affect the difference between the actual and target correlation functions. Now, we describe the new algorithm in detail below.

\section{Algorithm: Incremental Fourier Synthesis Method}

1) Create white-noise processes such that for $k_{x}=$ $0, \cdots, N$, and $k_{y}=0, \cdots, N / 2, \hat{W}\left(k_{x}, k_{y}\right) \sim$ $N(0,1), \hat{\phi}\left(k_{x}, k_{y}\right) \sim$ Uniform $[0,2 \pi)$, and $\phi(0,0)=$ $\phi(N / 2, N / 2)=\phi(N / 2,0)=\phi(0, N / 2)=0$. 
2) Calculate $R_{2}\left(m_{x}, m_{y}\right)$ (the desired correlation function of $I_{2}\left(m_{x}, m_{y}\right)$ ) by (8) for $m_{x}, m_{y}=0, \cdots, N / 2$, and symmetrically expand the correlation function via (12) and (13).

3) Calculate the power spectrum by

$$
\begin{aligned}
& \hat{R}_{2}\left(k_{x}, k_{y}\right) \\
& =\sum_{m_{x}=0}^{N-1} \sum_{m_{y}=0}^{N-1} R_{2}\left(m_{x}, m_{y}\right) e^{-j \frac{2 \pi k_{n} m_{x}}{N}} e^{-j \frac{2 \pi k_{y} m_{y}}{N}} .
\end{aligned}
$$

4) Define the actual positive semidefinite power spectrum as

$$
\hat{S}_{2}\left(k_{x}, k_{y}\right)= \begin{cases}0, & k_{x}=0 \text { or } k_{y}=0 \\ 0, & \text { if } \hat{R}_{2}\left(k_{x}, k_{y}\right)<0 \\ \hat{R}_{2}\left(k_{x}, k_{y}\right), & \text { otherwise }\end{cases}
$$

5) Synthesize the DFT coefficients of $I_{2}\left(m_{x}, m_{y}\right)$ by scaling the white noise from Step 1 as shown in the expression at the bottom of the page.

6) Calculate the second-order increments for $m_{x}, m_{y}=$ $0, \cdots, M-1$ as follows:

$$
\begin{aligned}
& I_{2}\left(m_{x}, m_{y}\right) \\
& \quad=\frac{1}{N^{2}} \sum_{k_{x}=0}^{N-1} \sum_{k_{y}=0}^{N-1} \hat{I}_{2}\left(k_{x}, k_{y}\right) e^{j \frac{2 \pi k_{x} m_{x}}{N}} e^{j \frac{2 \pi k_{y} m_{y}}{N}} .
\end{aligned}
$$

7) Create white noise processes such that for $k_{x}, k_{y}=$ $0, \cdots, N / 2, W_{x}\left(k_{x}\right) \sim N(0,1), W_{y}\left(k_{y}\right) \sim N(0,1)$, $\phi_{x}\left(k_{x}\right) \sim$ Uniform $[0,2 \pi), \phi_{y}\left(k_{y}\right) \sim$ Uniform $[0,2 \pi)$, and $\phi_{x}(0)=\phi_{y}(0)=\phi_{x}(N / 2)=\phi_{y}(N / 2)$.

8) Calculate $R_{x}\left(k_{x}, k_{y}\right)$ and $R_{y}\left(k_{x}, k_{y}\right)$ for $k_{x}, k_{y}=$ $0, \cdots, N / 2$ using (6) and (7). Symmetrically expand the correlation functions using (12) and (13).

9) Compute the desired power spectrum of the first-order increments at the zero frequencies via

$$
\begin{aligned}
& \hat{R}_{x}\left(k_{x}, 0\right)=\sum_{m_{x}=0}^{N-1} \sum_{m_{y}=0}^{N-1} R_{x}\left(m_{x}, m_{y}\right) e^{-j \frac{2 \pi k_{x} m_{x}}{N}} \\
& \hat{R}_{y}\left(0, k_{y}\right)=\sum_{m_{x}=0}^{N-1} \sum_{m_{y}=0}^{N-1} R_{y}\left(m_{x}, m_{y}\right) e^{-j \frac{2 \pi k_{y} m_{y}}{N}} .
\end{aligned}
$$

10) Define the actual positive semidefinite power spectrum of the first-order increments at the zero frequencies via the first case shown at the bottom of the next page.

11) Synthesize the DFT coefficients of the first-order increments by scaling white noise from Step 7, as shown in the second group of cases at the bottom of the next page.

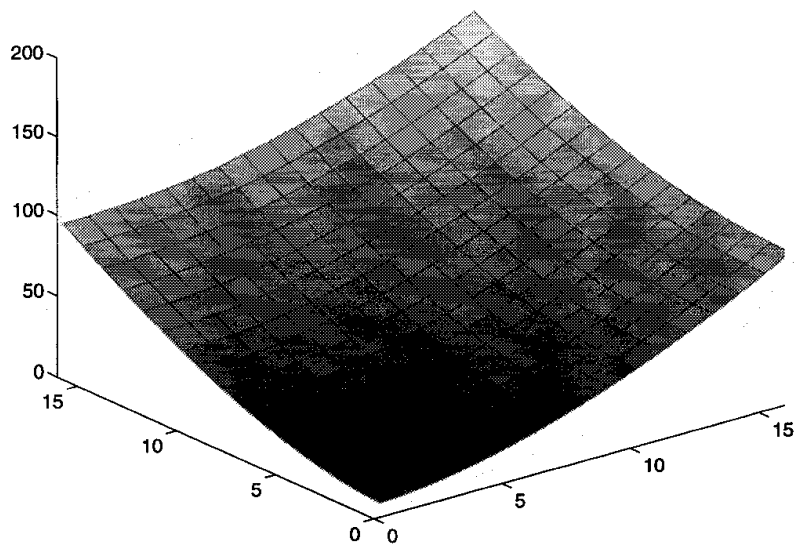

(a)

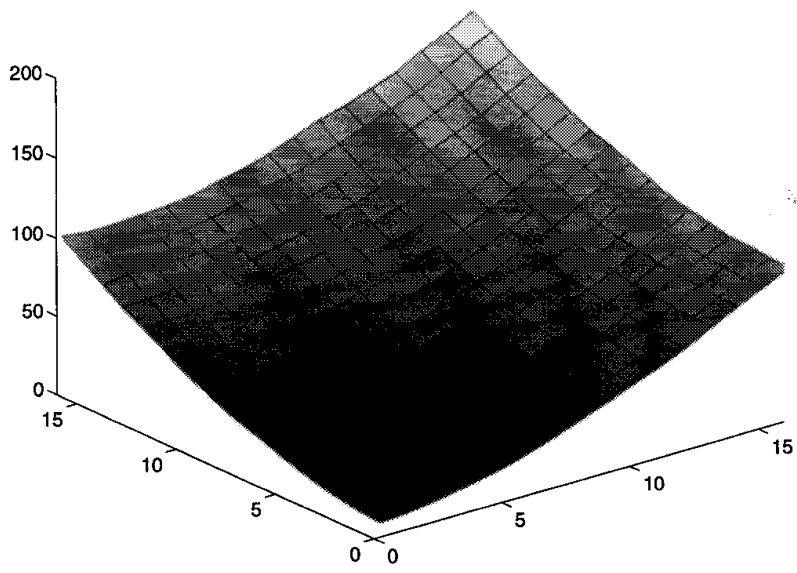

(b)

Fig. 2. Variance of the generated $\mathrm{fBm}$ images for $H=0.8$ : (a) Theoretical; (b) experimental.

12) Compute the first-order increments along the image boundaries for $m_{x}, m_{y}=0, \cdots, M-1$, as follows:

$$
\begin{aligned}
& I_{x}\left(m_{x}, 0\right)=\frac{1}{N^{2}} \sum_{k_{x}=0}^{N-1} \sum_{k_{y}=0}^{N-1} \hat{I}_{x}\left(k_{x}, k_{y}\right) e^{-j \frac{2 \pi m_{x} k_{x}}{N}} \\
& I_{y}\left(0, m_{y}\right)=\frac{1}{N^{2}} \sum_{k_{x}=0}^{N-1} \sum_{k_{y}=0}^{N-1} \hat{I}_{x}\left(k_{x}, k_{y}\right) e^{-j \frac{2 \pi m_{y} k_{y}}{N}} .
\end{aligned}
$$

13) Add up the increments to calculate the fBm field for $m_{x}, m_{y}=0, \cdots, M$ via

$$
\begin{aligned}
B(0,0) & =0 \\
B\left(m_{x}, 0\right) & =B\left(m_{x}-1,0\right)+I_{x}\left(m_{x}-1,0\right), \\
B\left(0, m_{y}\right) & =B\left(0, m_{y}-1\right)+I_{y}\left(0, m_{y}-1\right),
\end{aligned}
$$

$$
\hat{I}_{2}\left(k_{x}, k_{y}\right)= \begin{cases}N \sqrt{\hat{S}_{2}\left(k_{x}, k_{y}\right)} \hat{W}\left(k_{x}, k_{y}\right) e^{j \phi\left(k_{x}, k_{y}\right)}, & \text { for } k_{x}=0, \cdots, N-1 \text { and } k_{y}=0, \cdots, N / 2 \\ \hat{I}_{2}^{*}\left(N-k_{x}, N-k_{y}\right), & \text { for } k_{x}=0, \cdots, N-1 \text { and } k_{y}=N / 2+1, \cdots, N-1\end{cases}
$$




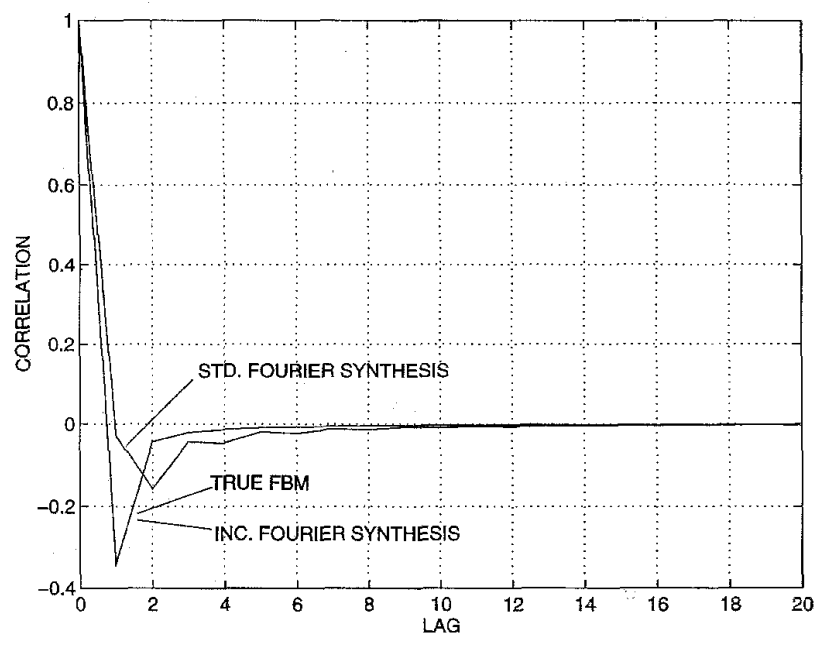

(a)

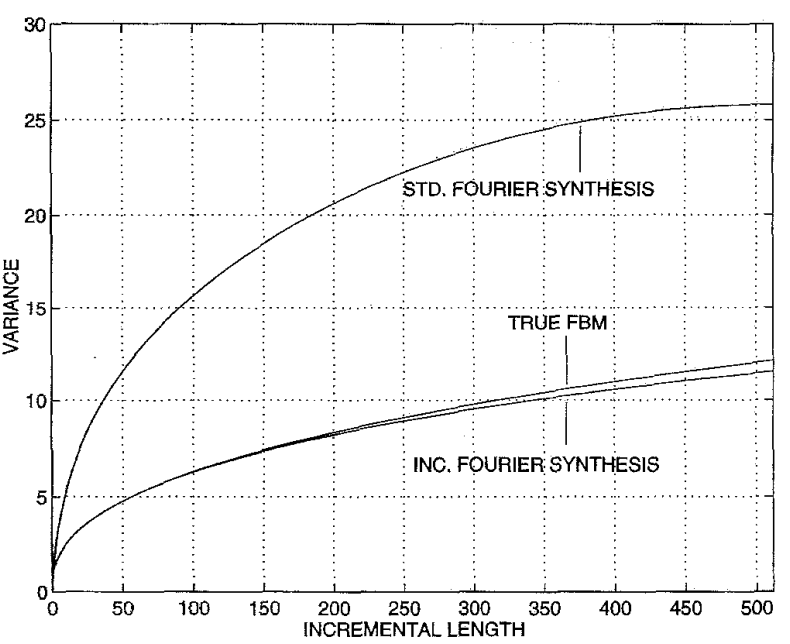

(b)

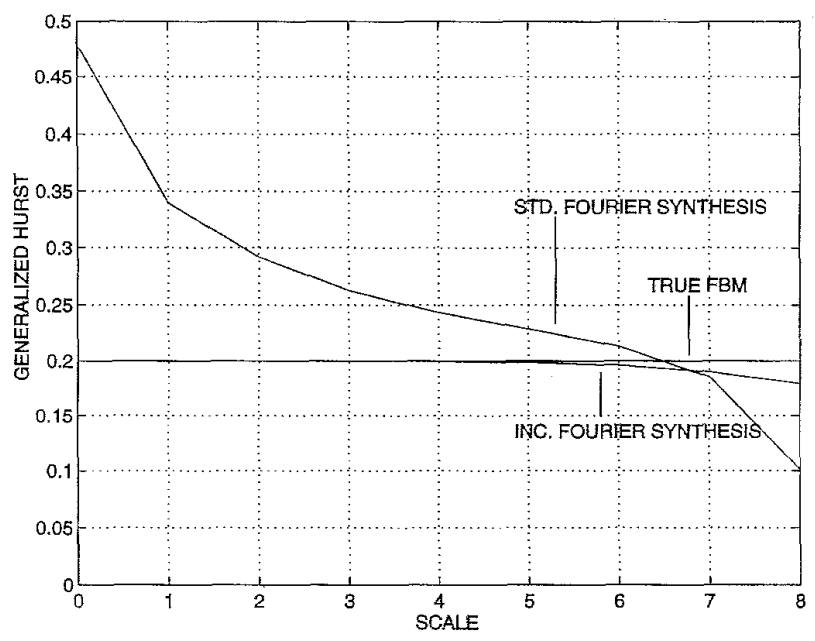

(c)

Fig. 3. Theoretical values for the statistics of the two Fourier synthesis methods and $\mathrm{Bm}$ given $512 \times 512$ realizations when $H=0.2$ : (a) Correlations: (b) structure functions; (c) generalized Hurst values.

$$
\begin{aligned}
B\left(m_{x}, m_{y}\right) & =B\left(m_{x}, m_{y}-1\right)+B\left(m_{x}-1, m_{y}\right) \\
& -B\left(m_{x}-1, m_{y}-1\right) \\
& +I_{2}\left(m_{x}-1, m_{y}-1\right)
\end{aligned}
$$

When implementing the algorithm, the low complexity of the FFT is exploited. To generate an $(M+1) \times(M+1)$ image, the algorithm uses $4(2 M+1) 1-D$ FFT's of size $2 M$ to compute (15) and (16) and another four FFT's of size $2 M$ to compute (17)-(20). In total, the algorithms requires $4 M+81-\mathrm{D}$ FFT's of size $2 M$. In contrast, the usual Fourier synthesis takes one 2-D inverse DFT that requires $2(M+1)$ 1-D FFT's. In other words, the incremental Fourier synthesis of $\mathrm{fBm}$ uses close to exact statistics of $\mathrm{fBm}$ with only twice the computational cost of standard Fourier synthesis.

\section{EXPERIMENTAL RESULTS}

We use the proposed incremental Fourier synthesis method to generated 256 realizations of $17 \times 17 \mathrm{fBm}$ processes with $H=0.8$ and $H=0.2$, where $\sigma^{2}=1$ for both cases. We calculated the variance at all pixels over the 256 independent images. Based on (4), the variance of pixel location $\left(m_{x}, m_{y}\right)$ should be ${\sqrt{m_{x}^{2}+m_{y}^{2}}}^{2 H}$. The theoretical and experimental variances are displayed in Figs. 1 and 2. Note that the usual

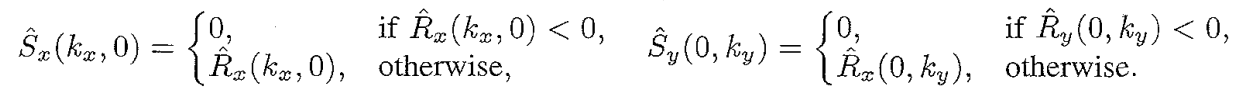




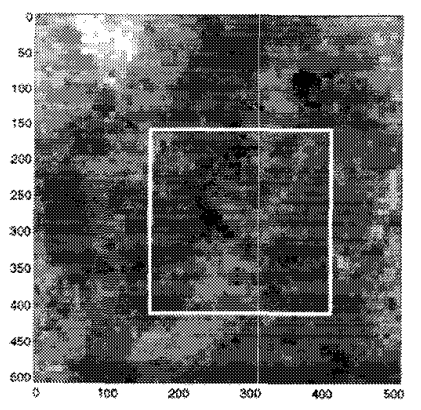

(a)

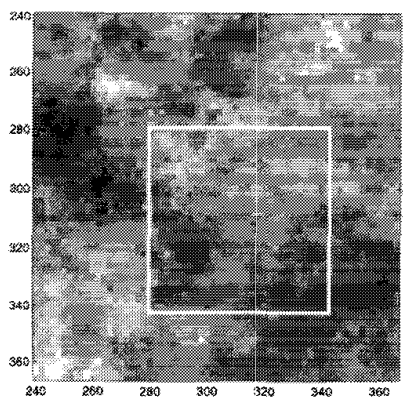

(c)

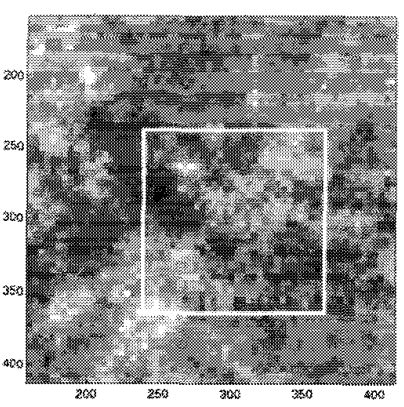

(b)

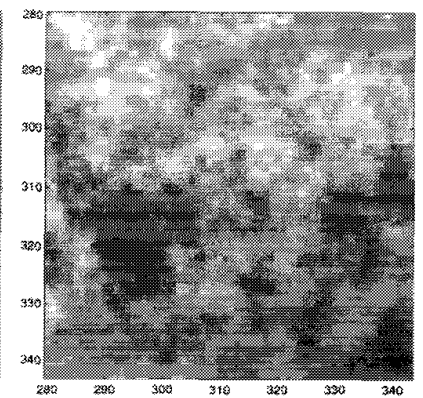

(d)

Fig. 4. Zooming into a texture generated by standard Fourier synthesis with $H=0.2$ : (a) Sampled every 8 units ( $s=3$ ); (b) sampled every 4 units $(s=2)$; (c) sampled every 2 units $(s=1)$; (d) sampled every unit $(s=0)$.

Fourier synthesis method generates a stationary process, and consequently, the variance is constant over all pixel values.

The theoretical statistics of images created by standard and incremental Fourier synthesis are compared in Fig. 3 for $512 \times 512$ images when $H$ is set to 0.2 . First, we calculate the correlations of the first-order increments by applying the inverse FFT to the power spectrums that were used to scale white noise. For instance, the actual correlation function for the process $I_{x}\left(m_{x}, m_{y}\right)$ generated by incremental Fourier synthesis is

$$
R_{x}^{a}\left(m_{x}, m_{y}\right)=\frac{1}{N^{2}} \sum_{k_{x}=0}^{N-1} \sum_{k_{y}=0}^{N-1} S_{x}\left(k_{x}, k_{y}\right) e^{j \frac{2 \pi k_{x} m_{x}}{N}} e^{j \frac{2 \pi k_{y} m_{y}}{N}}
$$

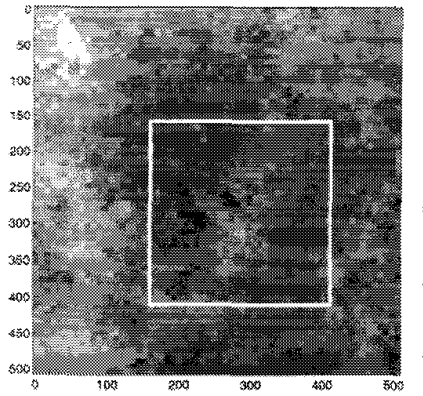

(a)

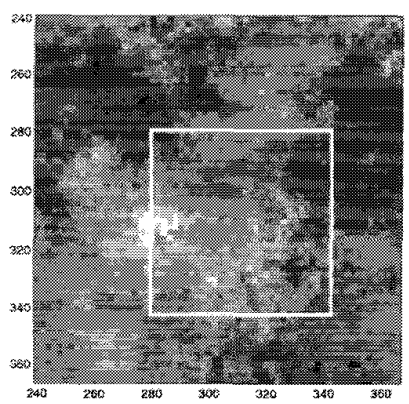

(c)

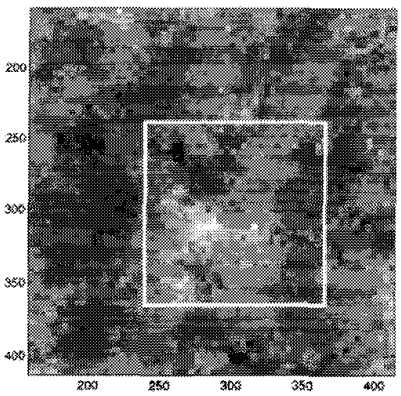

(b)

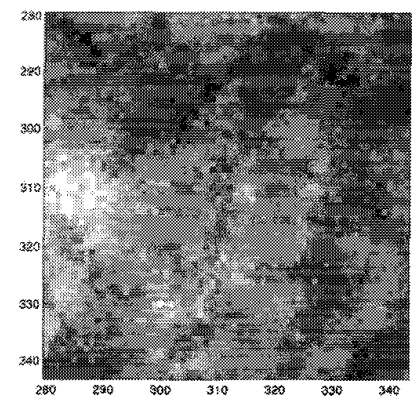

(d)
Fig. 5. Zooming into a texture generated by incremental Fourier synthesis with $H=0.2$ : (a) Sampled every 8 units; ( $s=3$ ); (b) sampled every 4 units $(s=2)$; (c) sampled every 2 units $(s=1)$; (d) sampled every unit $(s=0)$.

where

$$
S_{x}\left(k_{x}, k_{y}\right)=\frac{S_{2}\left(k_{x}, k_{y}\right)}{4 \sin ^{2}\left(\pi k_{y} / N\right)} \quad \text { for } k_{y} \neq 0
$$

and $S_{x}\left(k_{x}, 0\right)$ is given by Step 10 of the algorithm. Fig. 3(a) compares the correlations of both synthesis procedures with true $\mathrm{fBm}$. One can then calculate the theoretical values for the normalized variance as the displacement size of the $x$ directed increments of the generated picture grows, i.e.

$$
\tilde{f}(d)=\frac{\operatorname{VAR}\left[B\left(m_{x}+d, m_{y}\right)-B\left(m_{x}, m_{y}\right)\right]}{\operatorname{VAR}\left[B\left(m_{x}+1, m_{y}\right)-B\left(m_{x}, m_{y}\right)\right]}, \quad d \in \mathbf{Z}^{+}
$$

to be [4], as follows:

$\tilde{f}(d)=d+\frac{2}{R_{x}^{a}(0,0)} \sum_{l=1}^{d-1}(d-l) R_{x}^{a}(l, 0)$, for $d=1,2,3, \cdots$

$$
\begin{aligned}
& \hat{I}_{x}\left(k_{x}, k_{y}\right)= \begin{cases}-j \frac{\hat{I}_{2}\left(k_{x}, k_{y}\right)}{2 \sin \left(\pi k_{y} / N\right)} e^{-j \pi k_{y} / N}, & \text { for } k_{x}, k_{y}=1, \cdots, N-1, \\
N \sqrt{\hat{S}_{x}\left(k_{x}, 0\right)} \hat{W}_{x}\left(k_{x}\right) e^{j \phi_{x}\left(k_{x}\right),} & \text { for } k_{x}=0, \cdots, N / 2, \text { and } k_{y}=0, \\
\hat{I}_{x}^{*}\left(N-k_{x}, 0\right), & \text { for } k_{x}=N / 2+1, \cdots, N-1, \text { and } k_{y}=0 \\
0, & \text { otherwise. }\end{cases} \\
& \hat{I}_{y}\left(k_{x}, k_{y}\right)= \begin{cases}-j \frac{\hat{I}_{2}\left(k_{x}, k_{y}\right)}{2 \sin \left(\pi k_{x} / N\right)} e^{-j \pi k_{x} / N}, & \text { for } k_{x}, k_{y}=1, \cdots, N-1, \\
N \sqrt{\hat{S}_{y}\left(0, k_{y}\right)} \hat{W}_{y}\left(k_{y}\right) e^{j \phi_{y}\left(k_{y}\right),}, & \text { for } k_{x}=0 \text { and } k_{y}=0, \cdots, N / 2, \\
\hat{I}_{y}^{*}\left(0, N-k_{y}\right), & \text { for } k_{x}=0 \text { and } k_{y}=N / 2+1, \cdots, N-1, \\
0, & \text { otherwise. }\end{cases}
\end{aligned}
$$


TABLE I

Average Hurst Estimates FOR THE $64 \times 64$ IMAges

\begin{tabular}{|c||c|c|}
\hline & Fig. 4 & Fig. 5 \\
\hline (a) & 0.1952 & 0.1682 \\
\hline (b) & 0.2273 & 0.1756 \\
\hline (c) & 0.3020 & 0.2160 \\
\hline (d) & 0.3973 & 0.2064 \\
\hline
\end{tabular}

for incremental Fourier synthesis. The function $\tilde{f}(d)$ is known as the structure function. Since the defining feature of selfsimilar processes is a hyperbolic structure function (see (2) and (3)), the quality of a synthesis procedure is measured by how closely the structure function of its realizations match the desired hyperbolic function. For normal Fourier synthesis, the structure function is

$$
\tilde{f}(d)=\frac{R(0,0)-R(d, 0)}{R(0,0)-R(1,0)}
$$

where $R\left(m_{x}, m_{y}\right)$ is provided by the inverse DFT of $\hat{R}\left(k_{x}, k_{y}\right)$ as given by (14). Fig. 3(b) shows the structure functions for both synthesis procedures and $\mathrm{fBm}$. To better visualize the hyperbolic growth rate of $\tilde{f}(d)$, we plot the generalized Hurst parameters defined as

$$
\tilde{H}(s)=\frac{1}{2} \log _{2}\left(\tilde{f}\left(2^{s+1}\right) / \tilde{f}\left(2^{s}\right)\right)
$$

for both synthesis procedures and fBm in Fig. 3(c). The figure shows that the actual process generated by incremental Fourier synthesis has a nearly constant $\tilde{H}(s)$, i.e., virtually self-similar. Moreover, it is clear that images generated by standard Fourier synthesis are not statistically self-similar. In fact, the figure suggests that the generated images will be a little smoother at finer scales since the value of $H(s)$ becomes larger. Note that an analysis of the $y$-directed increments will yield the same results due to the isotropy of both generating algorithms.

To demonstrate the drawback of standard Fourier synthesis, we generated two realizations of $2-\mathrm{D} \mathrm{fBm}$ of size $512 \times 512$ using the two Fourier methods with $H=0.2$. Figs. 4 and 5 show the images generated by the standard and incremental Fourier methods, respectively, at different scales. At each scale, the resolution of the picture is $64 \times 64$, and each picture is scaled so that the dynamic range of the pixel values cover all 64 grey-level values. The statistical self-similarity is evident for the $\mathrm{fBm}$ realization created by our new method. As predicted by the generalized Hurst parameters, the $\mathrm{fBm}$ realization generated by traditional Fourier synthesis is a bit more blurred (or cloudy) at the finest scale as compared to the coarser scales. Moreover, we applied a wavelet-based fractal estimator presented in [5] to all horizontal and vertical strips for each of the $64 \times 64$ images. The average Hurst estimates are shown in Table I.

\section{CONCLUSIONS}

A new method called incremental Fourier synthesis was proposed to synthesize self-similar images based on a 2-D $\mathrm{fBm}$ model. The advantage of the method is that it is a relatively fast algorithm while it generates processes whose statistics virtually match those of true $\mathrm{fBm}$. Some interesting extensions to the algorithm can be investigated. For example, with the incremental Fourier method, one can also choose an arbitrary structure function and substitute the new $\tilde{f}(d)$ in (8), (6), and (7). By choosing alternative forms of the structure function, an artist has precise control of the "roughness" of the texture with respect to scale as shown in [6]. Furthermore, the algorithm can be extended to generate 3-D (video) and even higher dimension $\mathrm{fBm}$ at the expense of $O\left(N^{d} \log _{2}(N)\right)$ computations where $d$ is the dimension.

\section{REFERENCES}

[1] L. T. Bruton and N. R. Bartley, "Simulation of fractal multidimensional images using multidimensional recursive filters," IEEE Trans. Circuits Syst., vol. 41, pp. 181-188, Mar. 1994.

[2] S. Höfer, H. Hannachi, M. Pandit, and R. Kumaresan, "Isotropic two dimensional fractional Brownian motion and its application in ultrasonic analysis," in Proc. 14th IEEE Eng. Med. Biol. Soc. Conf., 1992, pp. $1267-1269$.

[3] S. Höfer, F. Heil, M. Pandit, and R. Kumaresan, "Segmentation of textures with different roughness using the model of isotropic twodimensional fractional Brownian motion," in Proc. IEEE ICASSP-93, vol. 5, Apr. 1993, pp. 53-56.

[4] L. M. Kaplan, "Fractal signal modeling: theory, algorithms, and applications," Ph.D. dissertation, Univ. of Southern California, Los Angeles, CA, 1994.

[5] L. M. Kaplan and C.-C. J. Kuo, "Fractal estimation from noisy data via discrete fractional Gaussian noise (dfGn) and the Haar basis," IEEE Trans. Signal Processing, vol. 41, pp. 3554-3562, Dec. 1993.

[6] _ "Texture roughness analysis and synthesis via extended selfsimilar (ESS) model," IEEE Trans. Pattern Anal. Machine Intell., vol. 17, pp. 1043-1056, Nov. 1995.

[7] J. P. Lewis, "Generalized stochastic subdivision," ACM Trans. Graphics, vol. 6, pp. 167-190, July 1987.

[8] B. B. Mandelbrot, The Fractal Geometry of Nature. San Francisco, CA: Freeman, 1982.

[9] B. B. Mandelbrot and J. W. Van Ness, "Fractional Brownian motions, fractional noises and applications," SIAM Rev., vol. 10, pp. 422-437, Oct. 1968.

[10] F. K. Musgrave, "Methods for realistic landscape imaging," Ph.D. dissertation, Yale Univ., New Haven, CT, 1993.

[11] H. O. Peitgen and D. Saupe, Eds. The Science of Fractal Images, New York: Springer-Verlag, 1988.

[12] W. Rümelin, "Fractal interpolation of random fields of fractional Brownian motion," in Fractal Geometry and Computer Graphics, J. L. Encatnacao, Ed. New York: Springer-Verlag, 1992.

[13] M. A. Stoksik, R. G. Lane, and D. T. Nguyen, "Practical synthesis of accurate fractal images," Graphic. Models Image Processing, vol. 57, pp. 206-219, May 1995.

[14] G. W. Wornell, "A Karhunen-Loëve-like expansion for $1 / f$ processes via wavelets," IEEE Trans. Inform. Theory, vol. 36, pp. 859-861, July 1990.

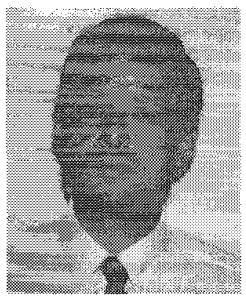

Lance M. Kaplan (M'95) received the B.S. degree with distinction from Duke University, Durham, NC, in 1989 and the M.S. and Ph.D. degrees from the University of Southern California (USC), Los Angeles, in 1991 and 1994, respectively, all in electrical engineering,

He held a National Science Foundation graduate fellowship and a USC Dean's Merit fellowship from 1990 to 1993 and worked as a research assistant in the Signal and Image Processing Institute at USC from 1993 to 1994. He joined the Reconnaissance Systems Department of the Hughes Aircraft Company in 1994. His research interests include fractal analysis, modeling and synthesis, image processing, multiresolution analysis, synthetic aperture radar, and remote sensing.

Dr. Kaplan is a member of Tau Beta Pi and Eta Kappa Nu. 


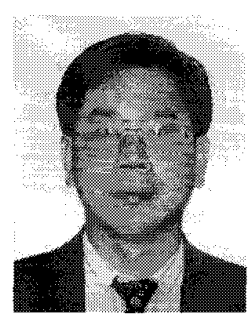

C.-C. Jay Kuo (SM'92) received the B.S. degree from the National Taiwan University, Taipei, Taiwan, in 1980 and the M.S. and Ph.D. degrees from the Massachusetts Institute of Technology, Cambridge, in 1985 and 1987, respectively, all in electrical engineering.

From October 1987 to December 1988, he was a Computational and Applied Mathematics (CAM) Research Assistant Professor in the Department of Mathematics at the University of California, Los Angeles. Since January 1989, he has been with the Department of Electrical Engineering Systems and the Signal and Image Processing Institute at the University of Southern California, Los Angeles, where he currently has a joint appointment as Associate Professor of Electrical Engineering and Mathematics. His research interests are in the areas of digital signal and image processing, video coding, wavelet theory and applications, multimedia technologies, and large-scale scientific computing. He has more than 180 technical publications appearing in journals and conferences.

Dr. Kuo is a member of Sigma Xi, SIAM, SPIE, and ACM. He serves as associate editor for the IEEE Transactions ON IMAGe Processing and the IEEE Transactions on Circuits and Systems for Video Technology. He is also on the editorial board of the JoURNAL OF VISUAL COMMUNICATION AND IMAGE REPRESENTATION. He was a recipient of the National Science Foundation Young Investigator Award in 1992 and Presidential Faculty Fellow Award in 1993. 